\title{
Deciphering the Origin of Ionized Gas in IC 1459 with VLT/MUSE
}

\author{
C. R. Mulcahey, ${ }^{1,4 \star}$ L. J. Prichard ${ }^{2} \dagger$ D. Krajnović ${ }^{3}$ and R. A. Jorgenson ${ }^{4}$ \\ ${ }^{1}$ Mount Holyoke College, 50 College St, South Hadley, MA 01075, USA \\ ${ }^{2}$ Space Telescope Science Institute, 3700 San Martin Drive, Baltimore, MD 21218, USA \\ ${ }^{3}$ Leibniz-Institute for Astrophysics, An der Sternwarte 16, 14482 Potsdam, Germany \\ ${ }^{4}$ Maria Mitchell Observatory, 4 Vestal Street, Nantucket, MA 02554, USA
}

Accepted XXX. Received YYY; in original form ZZZ

\begin{abstract}
IC 1459 is an early-type galaxy (ETG) with a rapidly counter-rotating stellar core, and is the central galaxy in a gas-rich group of spirals. In this work, we investigate the abundant ionized gas in IC 1459 and present new stellar orbital models to connect its complex array of observed properties and build a more complete picture of its evolution. Using the MultiUnit Spectroscopic Explorer (MUSE), the optical integral field unit (IFU) on the Very Large Telescope (VLT), we examine the gas and stellar properties of IC 1459 to decipher the origin and powering mechanism of the galaxy's ionized gas. We detect ionized gas in a non-disk-like structure rotating in the opposite sense to the central stars. Using emission-line flux ratios and velocity dispersion from full-spectral fitting, we find two kinematically distinct regions of shocked emission-line gas in IC 1459, which we distinguished using narrow $\left(\sigma \leq 155 \mathrm{~km} \mathrm{~s}^{-1}\right)$ and broad $\left(\sigma>155 \mathrm{~km} \mathrm{~s}^{-1}\right)$ profiles. Our results imply that the emission-line gas in IC 1459 has a different origin than that of its counter-rotating stellar component. We propose that the ionized gas is from late-stage accretion of gas from the group environment, which occurred long after the formation of the central stellar component. We find that shock heating and AGN activity are both ionizing mechanisms in IC 1459 but that the dominant excitation mechanism is by post-asymptotic giant branch stars from its old stellar population.
\end{abstract}

Key words: galaxies: individual: IC 1459, - galaxies: elliptical, - galaxies: evolution, galaxies: kinematics and dynamics, - galaxies: nuclei, - galaxies: structure

\section{INTRODUCTION}

Early-type galaxies (ETGs) are typically characterised as quiescent, having an old stellar population, little gas and dust, and being devoid of spiral arms and structure. A feature unique to ETGs are kinematically distinct cores (KDCs), which occur when there is a rapid change of the position angle of the kinematic axis of more than $30^{\circ}$ and the rotational velocity approaches zero in the transition region (Krajnović et al. 2011). The first detection of KDCs in ETGs was made by Efstathiou et al. (1982) using long-slit spectroscopy to study stellar kinematics. Subsequent studies used a similar approach and detected additional KDCs (Bender 1988; Carollo \& Danziger 1994; Franx \& Illingworth 1988; Jedrzejewski \& Schechter 1988). Long-slit spectroscopy can be costly for large galaxy samples and only produces one spectrum per galaxy from which physical properties of the whole system are inferred. The advent of wide-field, high-resolution integral field spectroscopy (IFS) has revolutionised our understanding of ETGs by enabling more detailed investigations (see Cappellari 2016 for a review). Instead of obtaining one spectrum of light per galaxy, integral field units (IFUs) allow a galaxy



$\dagger$ E-mail: lprichard@stsci.edu
}

to be viewed in three dimensions (3D), where every spatial pixel (spaxel) across the surface of a galaxy contains a full spectrum of light.

The SAURON (Bacon et al. 2001) survey (de Zeeuw et al. 2002) was the first IFS study to spatially resolve stellar kinematics, ionized gas, and stellar populations, and did so for a sample of 48 nearby ETGs. Their spatially resolved kinematics (Emsellem et al. 2004) qualitatively separated ETGs into two classes: irregular, slow rotating, high-mass $\left(\mathrm{M}_{\text {crit }} \gtrsim 2 \times 10^{10} \mathrm{M}_{\odot}\right)$ ETGs $(\sim 15 \%$ of population) and regular, fast rotating, low-mass ETGs (Emsellem et al. 2007), which resemble disk models (Krajnović et al. 2008). The ATLAS $^{3 \mathrm{D}}$ survey (Cappellari et al. 2011) combined IFS data with theoretical modelling, which led to additional sub-classifications of slow rotators (Krajnović et al. 2011). Although KDCs are rare in ETGs $(\sim 7 \%)$, they are relatively common in slow rotators $(\sim 42 \%$; Krajnović et al. 2011).

The stellar and gas kinematics from each spaxel of IFS data provides a wealth of information that can be used to understand a galaxy's inherent properties and evolution in greater depth. Investigating stellar and gas components in isolation reveals unique evolutionary processes and is made possible by developments in full-spectral fitting codes and supporting spectral libraries and stellar population synthesis models (e.g., Cappellari \& Emsellem 2004; 
Cappellari 2017; Vazdekis et al. 2010, 2016). Studying the stellar component, for example, discerns whether a galaxy has been constructed from gas-poor or gas-rich merging events (e.g., Cappellari et al. 2013; Emsellem et al. 2011; Naab et al. 2014). However, the stellar component does not reveal the complete evolutionary history of a galaxy because a notable percentage of ETGs exhibit ionized gas in the emission-lines of optical spectra (40-80\%; e.g., Caldwell 1984; Phillips et al. 1986; Kim 1989; Buson et al. 1993; Goudfrooij et al. 1994; Macchetto et al. 1996; Sarzi et al. 2006, 2010).

Emission-line gasses reveal how gas accretion or gas-rich mergers have influenced the assembly of the galaxy (e.g., BarreraBallesteros et al. 2015; Davis et al. 2011; Sarzi et al. 2006) and can be used to understand the mechanisms behind star formation (SF; e.g., Alatalo et al. 2011; Cheung et al. 2016; Ho et al. 2016). Emission-line ratios, which are used extensively in the BaldwinPhillips-Terevich (BPT) diagrams (Baldwin et al. 1981; Veilleux \& Osterbrock 1987) and the WHAN (equivalent width of $\mathrm{H} \alpha$ versus $[\mathrm{NII}] / \mathrm{H} \alpha$ ) diagram (Cid Fernandes et al. 2011), are key diagnostics used to estimate whether the principal power source of galaxies is from $\mathrm{SF}$ or from the central active galactic nucleus (AGN). With IFU data, the emission-line ratios of these diagrams can be spatially resolved, revealing multiple ionization mechanisms across the surface of a galaxy. Moreover, IFU data can unveil extended shocks from outflows, galactic winds, and tidally induced shocks (e.g., Monreal-Ibero et al. 2006, 2010; Farage et al. 2010; Rich et al. 2010, 2011).

To hone our understanding of the formation and evolution of galaxies with KDCs, we investigate IC 1459, a prototypical, massive (M 4-6 $\times 10^{11} M_{\odot}$; Cappellari et al. 2002; Samurović \& Danziger 2005) ETG with a counter-rotating core. IC 1459 is a local ( $D=30.3$ $\pm 4.0 \mathrm{Mpc}$; Ferrarese \& Merritt 2000), bright $\left(\mathrm{M}_{\mathrm{v}} \simeq-22.3\right.$; Paturel et al. 1997) E3 ETG and it is the central galaxy of a gas-rich group of 11 galaxies, which mainly consists of spirals (group number 15; Huchra \& Geller 1982; Serra et al. 2015). The galaxy shows clear evidence for past group interactions like tidal features in deep images (Malin 1985) and shells at large radii (Forbes et al. 1995). The outer regions of the galaxy and central ionized gas component rotate in one direction at $\mathrm{V}_{\max } \sim 45 \pm 8 \mathrm{~km} \mathrm{~s}^{-1}$ while the central stellar component rapidly counter rotates at $\mathrm{V}_{\max } \sim 170 \pm 20 \mathrm{~km}$ $\mathrm{s}^{-1}$ (Franx \& Illingworth 1988).

IC 1459 is a gas-rich ETG containing molecular gas (Prandoni et al. 2012), and ionized gas in its core (e.g., Franx \& Illingworth 1988; Phillips et al. 1986). Neutral HI gas, which was most likely stripped away from NGC 7418 through tidal interactions (Oosterloo et al. 2018; Iodice et al. 2020), is abundant in the intra-group environment (Serra et al. 2015; Saponara et al. 2018). IC 1459, itself, does not contain HI gas (Serra et al. 2015), though the galaxy's ionized gas can be linked to the surrounding HI clouds (Saponara et al. 2018). IC 1459 hosts a bright radio source (PKS 2254-367; Tingay \& Edwards 2015) and Cappellari et al. (2002) measured the mass of the central black hole to be between $\sim 3.5 \times 10^{8} \mathrm{M}_{\odot}$ (from gas kinematics) and $2.6 \pm 1.1 \times 10^{9} \mathrm{M}_{\odot}$ (from stellar kinematics). The galaxy is also characterised as a Low Ionisation Nuclear Emission-line Region (LINER; Phillips et al. 1986; Verdoes Kleijn et al. 2000; Annibali et al. 2010).

A recent optical (MUSE: Multi-Unit Spectroscopic Explorer, Bacon et al. 2010) and infrared (KMOS: K-band Multi-Object Spectrograph, Sharples et al. 2013) IFU study of IC 1459 investigated the evolution of the galaxy focusing on the stellar populations, initial mass function (IMF), and stellar kinematics (Prichard et al. 2019). The stellar velocity dispersion revealed that IC 1459 is dynamically hot along the major axis, which is indicative of counter-rotating stel- lar components extending throughout the galaxy beyond the core. The stars show a radially flat and bottom-heavy IMF and old stellar ages throughout the galaxy, further supporting the theory that it comprises smooth co-spatial counter-rotating populations. However, orbital modelling is needed to fully understand if this is the case for IC 1459. We know that the counter-rotating stellar component formed at early times and therefore the stars alone do not give the full evolutionary picture of this gas-rich ETG. The origin of IC 1459's abundant gas remains unknown and the excitation mechanisms of its ionized gas is undetermined. We are also yet to understand the relationship, if any, between the gaseous and stellar components of the galaxy as they exist today.

To help us understand the evolutionary history of IC 1459, we build upon the work of Prichard et al. (2019) and use the existing MUSE data to study the galaxy's ionized gas in detail. We also construct dynamical models to investigate the internal orbital structure, the nature of the KDC, and its potential link with the emissionline gas. This paper is organised as followed: Section 2 outlines the MUSE observations and data reduction, Section 3 details the spectroscopic analysis of the MUSE cube. In Section 4 we present the resulting kinematic maps of IC 1459's ionized gas, Section 5 details new orbital models for the stellar population in IC 1459, and we discuss and conclude our findings in Section 6.

\section{OBSERVATIONS AND DATA REDUCTION}

The MUSE data used in this work is the same cube presented and prepared as described in Prichard et al. (2019). We briefly summarise the observations and reduction of the MUSE cube here. MUSE is mounted on the European Southern Observatory's (ESO's) Very Large Telescope (VLT) in Paranal, Chile. It is a large field-of-view (FOV; $\left.1^{\prime} \times 1^{\prime}\right)$ panchromatic optical $(\sim 4600-9300 \AA$ A) instrument with a Wide Field Mode (WFM) resolving power ranging $\mathrm{R}=1770-3590$ and a pixel scale of $0.2^{\prime \prime}$. The MUSE data of IC 1459 were taken October 14, 2014 (program ID 094.B-0298(A), P.I. C. J. Walcher) with total integration time of $368 \mathrm{~s}$ and seeing 1.3-1.6" using the MUSE_wfmnoao_obs_genericoffset observing template. The MUSE observations were taken during bright time (high Moon illumination) so have increased photon noise.

Reduced MUSE data is accessible on the ESO Science Archive $^{1}$. The data were reduced using the MUSE-1.6.1 pipeline and were published as a reduced data cube on the $22^{\text {nd }}$ June 2016. The reduced MUSE data cube of IC 1459 still contained strong contamination from bright sky lines. An additional sky subtraction was executed by subtracting a spectrum from the edge of the MUSE cube, that was dominated by sky, from the rest of the spaxels ${ }^{2}$. The cube was then cropped to remove regions strongly affected by sky and that had low signal-to-noise $(\mathrm{S} / \mathrm{N})$. The resulting cube is the central $30^{\prime \prime} \times 30^{\prime \prime}, 150 \times 150$ pixels centred on IC 1459's core. Residual sky features are still visible across the cube, especially in the red end of the spectra as demonstrated in Figure 1. For our analyses of the gas emission (Section 3.2), we use the bluest part of the spectrum, least affected by residual sky features, and masked bright sky lines.

\footnotetext{
1 http://archive.eso.org/cms.html

2 The subtraction of the pseudo sky and cube cropping was done by Joshua Warren and is described in his DPhil thesis.
} 


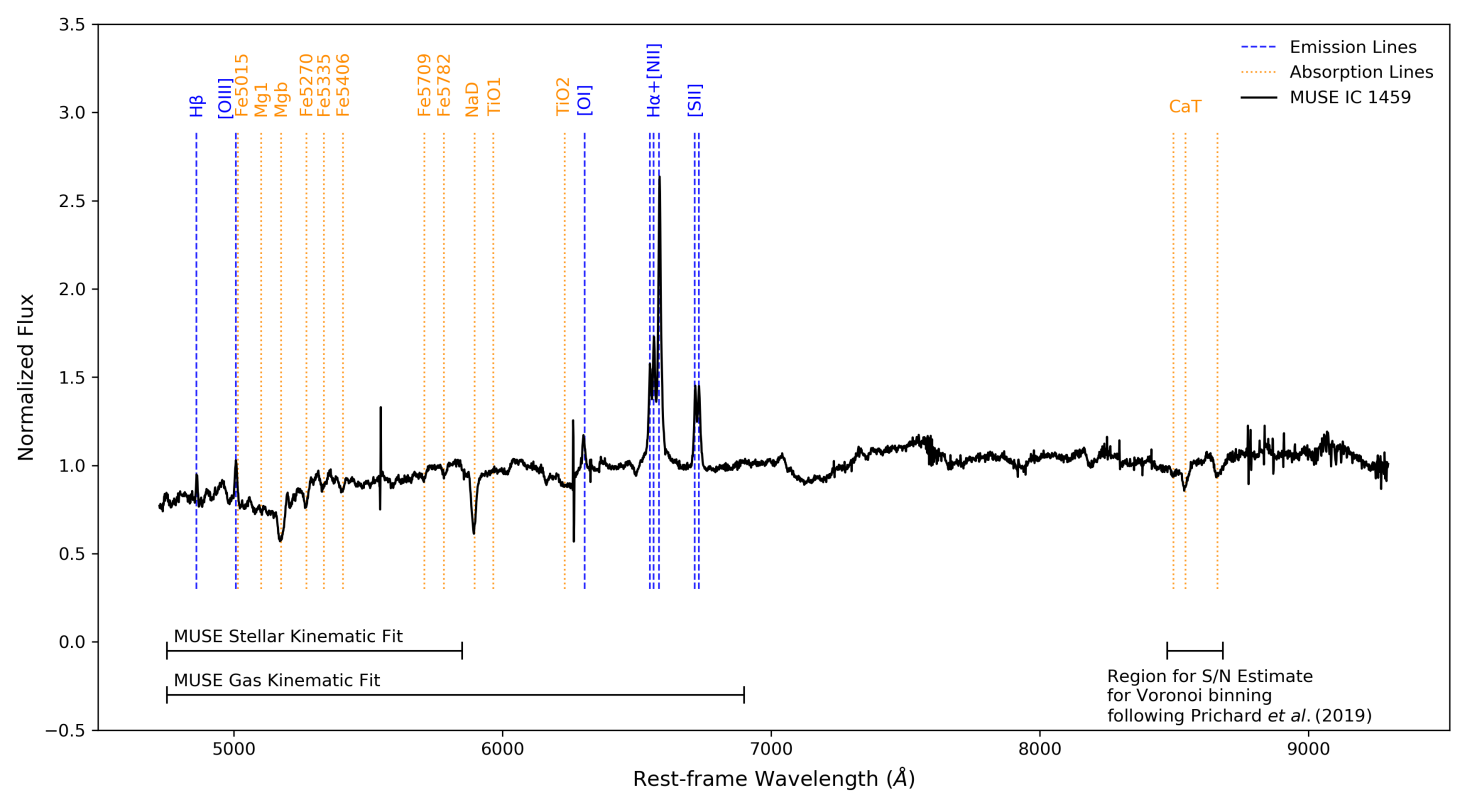

Figure 1. Spectrum extracted from the central spaxel of IC 1459. Gas emission lines (blue dashed lines) and stellar absorption lines (orange dotted lines) are shown. All unlabelled spectral features are sky-line contamination. Below the spectrum, we indicate the kinematic fit ranges for extracting gas and stellar kinematics, and the region where conservative S/N values were estimated for Voronoi binning in-line with Prichard et al. 2019. See Section 3.1.

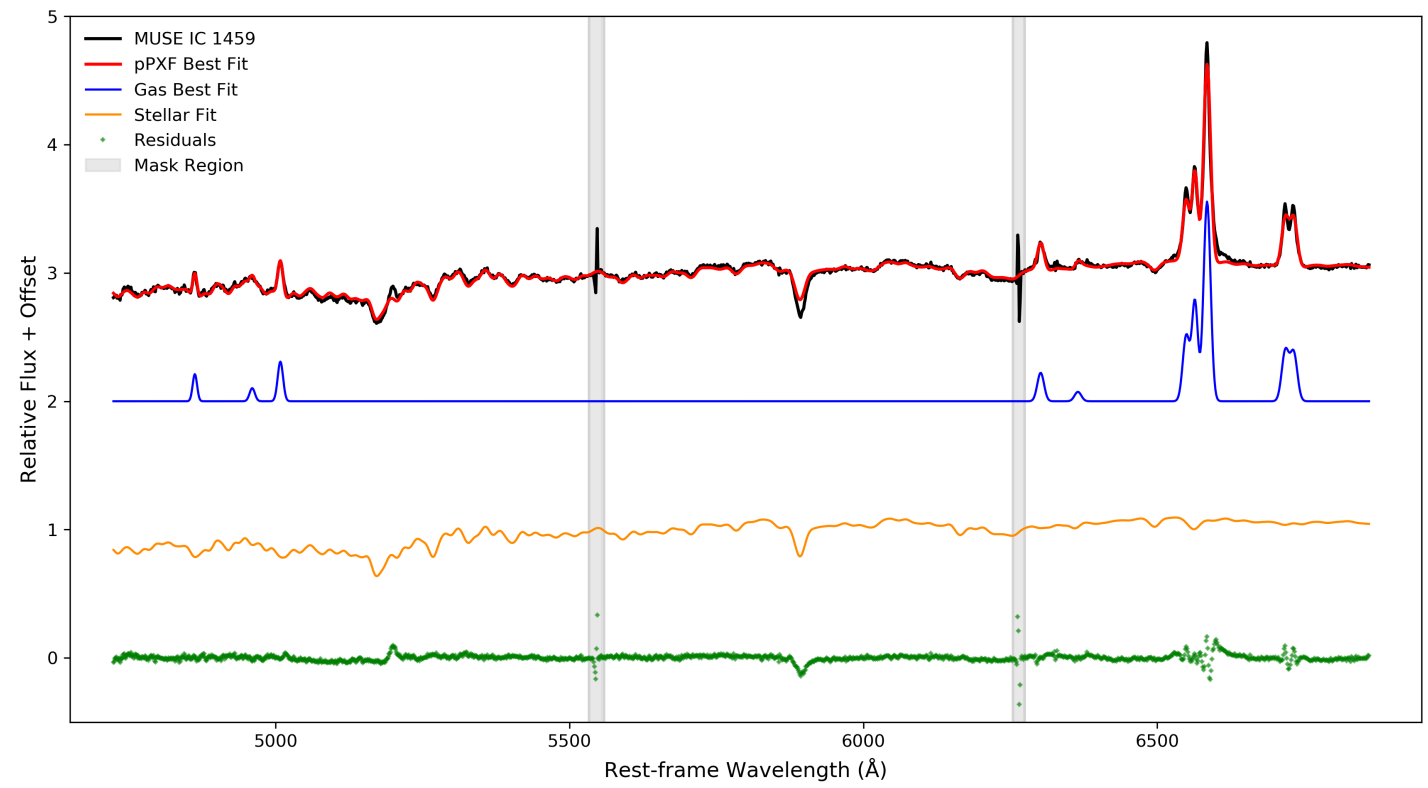

Figure 2. pPXF fit to the spectrum of the central spaxel of IC 1459 over the gas kinematics fitting region. The MUSE spectra (black), best fit (red), gas emission component (blue), stellar component (orange), and residuals (green) are shown. The grey shaded regions are locations of bright sky lines that were masked. 


\section{DATA ANALYSIS}

\subsection{Voronoi Binning}

Spectra from the MUSE data cube were used to measure the stellar and gas kinematics of IC 1459 (Figure 2). Because the quality and $\mathrm{S} / \mathrm{N}$ of the spectra varied across the MUSE cube, we used the Voronoi binning method of Cappellari \& Copin (2003) that adaptively bins data into regions of constant $\mathrm{S} / \mathrm{N}$ ratio. The VORONOI_2D_BINNING ${ }^{3}$ software needed a signal and noise for each spaxel over the MUSE cube. To get initial estimates of the S/N of each spectrum for creating the Voronoi bins, we opted to use the calcium triplet (CaT), as used in Prichard et al. (2019), so that the results of the two studies could be accurately compared. We also required the larger bins and smoother variations for the stellar orbital modelling presented in Section 5. As we fit both the stars and gas within each bin, we wanted to accurately compare both components across the galaxy by extracting their properties within the same bins. The $\mathrm{S} / \mathrm{N}$ values were determined by fitting the continuum region of the CaT (as defined by Cenarro et al. 2001). Signal values were estimated from the average continuum level around the $\mathrm{CaT}$ and noise values were estimated from the standard deviation of these continuum data. The MUSE cube was binned to $\mathrm{S} / \mathrm{N}=20$, which produced 4329 unique bins.

Although the CaT feature is in the noisier red end of the spectrum and the binning is therefore more conservative than using the bluer end of the spectrum, the resulting bins have only one or two spaxel size at the centre of the galaxy and these get progressively larger towards the lower S/N outskirts as expected, so we deemed these bins more than sufficient for our analysis of the resolved properties of the gas. To ensure that using the CaT feature rather than a cleaner feature toward the blue end of the spectrum did not affect our results, we also measured the $\mathrm{S} / \mathrm{N}$ from continuum regions around $\mathrm{Mg} b$. We found that the kinematics and results extracted from the $\mathrm{Mg} b$ bins were the same, and that the only difference was the finer bin sizes for the same $\mathrm{S} / \mathrm{N}$ threshold as CaT. Increasing the $\mathrm{Mg} b \mathrm{~S} / \mathrm{N}$ binning threshold to produce roughly the same number of bins as for $\mathrm{CaT}$ produced a comparable bin distribution. As we required the coarser bins anyway for the orbital modelling, we stuck with using the CaT feature so we could directly compare results with Prichard et al. (2019). Normalising and taking the median of the spectra in each Voronoi bin produced spectra to analyse above a threshold $\mathrm{S} / \mathrm{N}$ value across the entire MUSE cube.

\subsection{Extracting Kinematics}

We used the Penalized PiXel-Fitting algorithim $\left(\mathrm{pPXF}^{4}\right.$, developed by Cappellari \& Emsellem 2004; Cappellari 2017) to calculate the line-of-sight-velocity distribution (LOSVD) of the stellar and gas components of IC 1459. To extract the stellar and gas kinematics from the MUSE cube with pPXF, we used both stellar and gas emission-line templates, which are parametrised using Gauss-Hermite functions (Cappellari 2017) . We used the Mediumresolution Isaac Newton Telescope Library of Empirical Spectra (MILES; Sánchez-Blázquez et al. 2006; Vazdekis et al. 2010) ${ }^{5}$ library of stellar model templates to fit the average spectrum in each Voronoi bin.

pPXF was run on the normalised and averaged spectrum within

\footnotetext{
3 http://www-astro.physics.ox.ac.uk/ mxc/software/

4 Publicly available from http://purl.org/cappellari/software

5 The MILES library is available from http://miles.iac.es/.
}

each Voronoi bin across the cube. All spectra from MUSE and templates were logarithmically rebinned with flux conserved prior to fitting. As recommended for pPXF in Cappellari (2017), to extract the best possible fits of the stellar and gas components, we fit the spectra in two stages and the $\chi^{2}$ was minimised to find the best fit. We fitted the stellar and gas components simultaneously but used different fitting regions and input parameters optimised to cleanly extract information from each.

We created a noise spectrum for each bin by normalising and median combining the squared and rooted noise spectra from the error cube. We then scaled this combined error spectrum to a more accurate measure of the $\mathrm{S} / \mathrm{N}$ over the stellar fitting region by doing an initial run of pPXF to measure the "residual noise" from each binned spectrum. We fitted the spectrum and subtracted the best fit from the data to get the residuals. We then measured residual noise by taking the outlier-resistant standard deviation $(1.4826 \times$ median absolute deviation or MAD_STD) and the signal by taking the median of each spectrum over the fitting region to determine a signal-to-residual noise $(\mathrm{S} / \mathrm{rN})$ value for each bin. The error spectra were accurately scaled to the $\mathrm{S} / \mathrm{rN}$ of the fitting region determined for each bin, these values ranged from $\sim 70$ in the central bright pixel out to the limiting binning threshold in the outer region of $\sim$ 20 . The $\mathrm{S} / \mathrm{rN}$ scaled error spectra were logarithmically rebinned and used as inputs to pPXF for the stellar and gas kinematic fits.

To extract stellar kinematics, we used pPXF to fit the $\sim 4750$ $5850 \AA$ interval for the stellar component, as it is free from strong gas emission lines and contains the strongest stellar absorption features with minimal sky emission. We masked one strong sky-line region between $\sim 5570-5587 \AA$. We fit the spectrum using six moments of the LOSVD $\left(V, \sigma, h_{3}, h_{4}, h_{5}, h_{6}\right)$ and used additive polynomials of order 10 to extract the stellar kinematics.

The gas kinematics were then fitted separately with pPXF where the previously measured stellar kinematics $(V, \sigma)$ from the corresponding Voronoi bin were held fixed for the stellar templates only. This reduced the number of free parameters when freely fitting the gas emission templates and is as recommended in Cappellari (2017). To extract the gas kinematics, we fitted over the $\sim 4750$ $6900 \AA$ interval and excluded regions affected by strong sky-line features ( 5570-5587 $\AA, \sim 6295-6305 \AA$ ) using pPXF's goodpixels feature. We constructed a set of Gaussian gas emission-line templates to fit the gas emission lines in our wavelength range $(\mathrm{H} \beta$, $\mathrm{H} \alpha,[\mathrm{SII}] \lambda 6731,6731,[\mathrm{OIII}] \mathrm{d} \lambda 5007,[\mathrm{OI}] \_\mathrm{d} \lambda 6300,[\mathrm{NII}] \mathrm{d}$ 16583). We specified the Balmer series to be input as separate templates (tie_balmer=False) and the [OIII] and [SII] doublet ratios to be limited in-line with theory (limit_doublets=True). The [OI], [OIII] and [NII] doublets (*_d) use the fixed theoretical flux ratios, so only the stronger of the two lines are fitted. We fitted the gas emission lines with two moments of $\operatorname{LOSVD}(V, \sigma)$ and used multiplicative polynomials of order 4 .

The uncertainties of the stellar and gas LOSVD moments were calculated by performing Monte Carlo simulations for each bin. The original spectrum was perturbed 50 times, using random values selected from a standard normal Gaussian distribution (mean 0, variance 1) multiplied by each wavelength pixel of the S/rN-scaled error spectrum, to create 50 bootstrap spectra for each bin. We repeated the pPXF fits using the same input parameters for the gas and stellar fits respectively for each of the bootstrap spectra. The errors on the stellar and gas LOSVD parameters were then derived using the outlier-resistant MAD_STD of the values from the fits of the 50 bootstrap spectra. The stellar kinematic maps and their errors were used for the orbital modelling described in Section 5. 

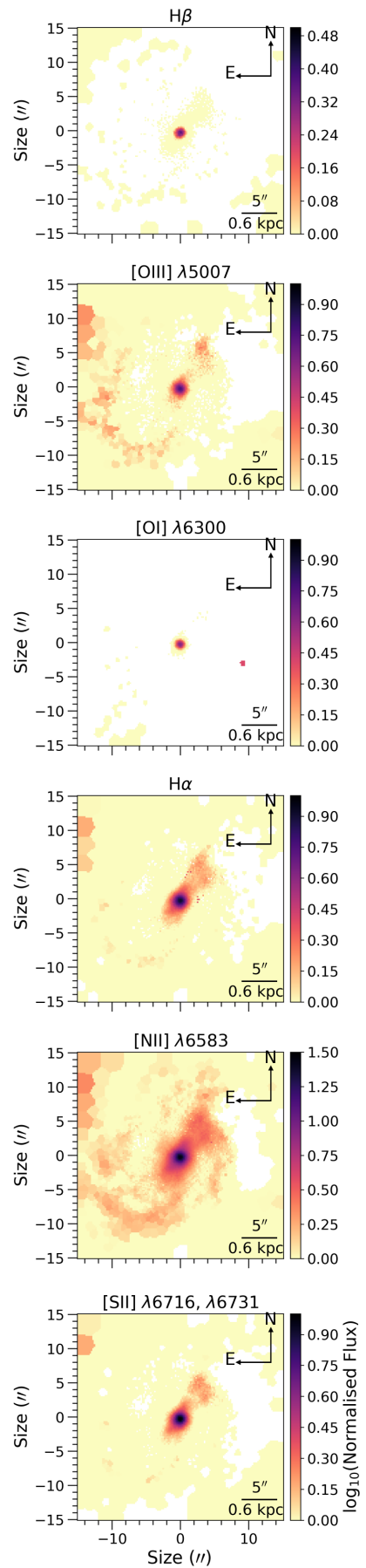
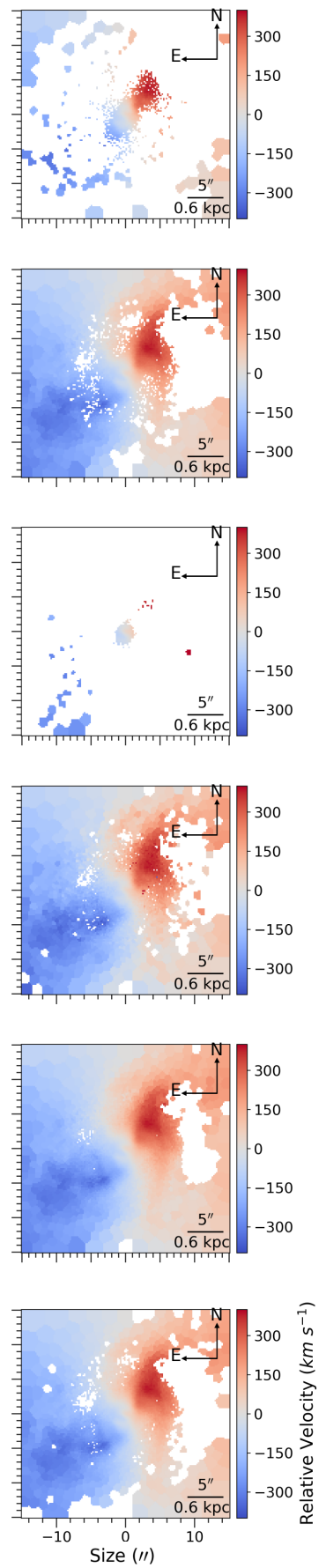
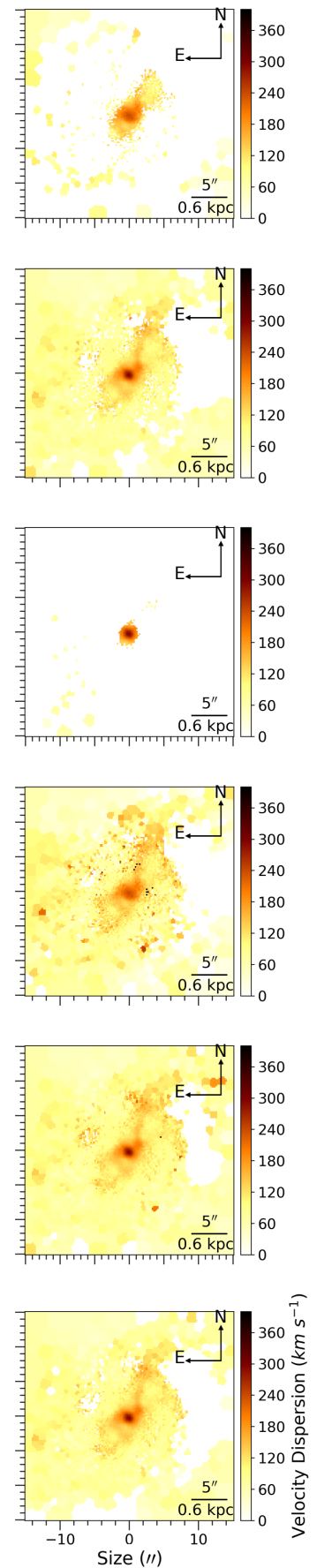
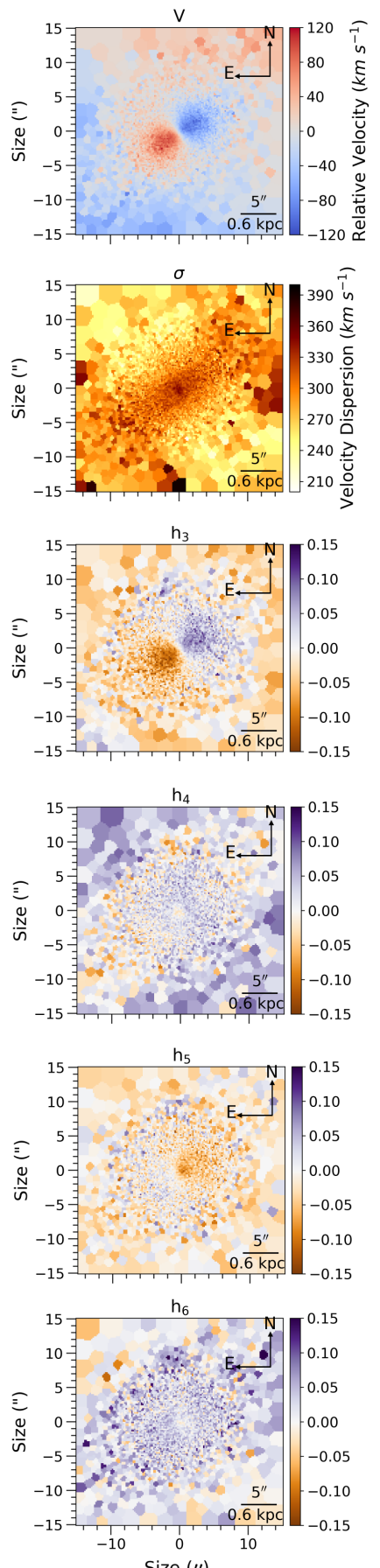

Figure 3. Left of vertical black line: Maps of normalised emission-line flux (left column), relative velocity (middle column), velocity dispersion (right column) for all bins with $\mathrm{A} / \mathrm{rN}>3$ for each feature as measured with pPXF. The emission-line features from top to bottom are: $\mathrm{H} \beta$, [OIII]_d $\lambda 5007$, H $\alpha$, [NII]_d $\lambda 6583$, [OI]_d $\lambda 6300$, and [SII] 16731, 6731. Right of vertical black line: Maps of the stellar LOSVD moments of IC 1459 as measured with pPXF. Top to bottom: stellar relative velocity, velocity dispersion, Gauss-Hermite coefficient $h_{3}, h_{4}, h_{5}$, and $h_{6}$.

\section{THE IONIZED GAS OF IC 1459}

\subsection{Maps of Ionized Gas in IC 1459}

We present spatially resolved kinematic and flux maps of the emission-line gasses in Figure 3. Only those bins with an amplitude- to-residual noise ratio $(\mathrm{A} / \mathrm{rN})>3$ are plotted (following Sarzi et al. 2006). The kinematic maps in the second column of Figure 3 indicate that the gas is blue shifted with a maximum relative velocity of $-344 \mathrm{~km} \mathrm{~s}^{-1} \pm 9$ and red shifted with a maximum relative velocity of $389 \mathrm{~km} \mathrm{~s}^{-1} \pm 7$. In the very centre of the galaxy $\left(2^{\prime \prime}\right)$, the rotation of gas is disordered and dispersion dominated. At larger radii the 


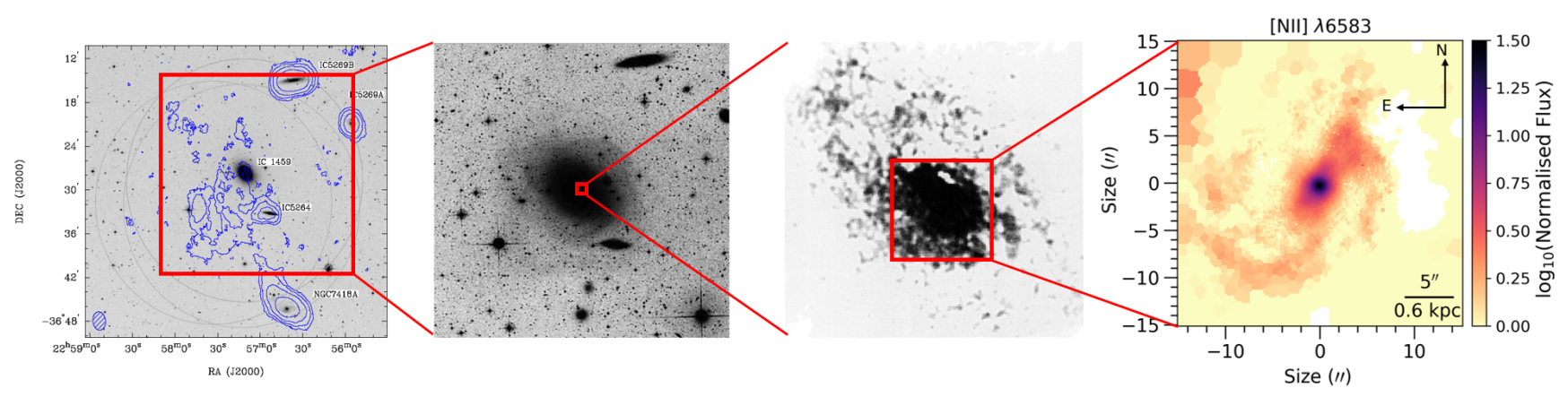

Figure 4. Images of IC 1459 at progressively smaller radii. From left to right: HI map of the IC 1459 group ( 40 across) from Saponara et al. 2018, deep optical image of IC $1459\left(\sim 28^{\prime}\right.$ across) from Malin 1985, H $\alpha+[\mathrm{NII}]$ image of IC $1459\left(\sim 1^{\prime} .34\right.$ across) from Goudfrooij et al. 1990, and the flux map of [NII] that we present in this study ( $30^{\prime \prime}$ across). North is up and east is left in all images.
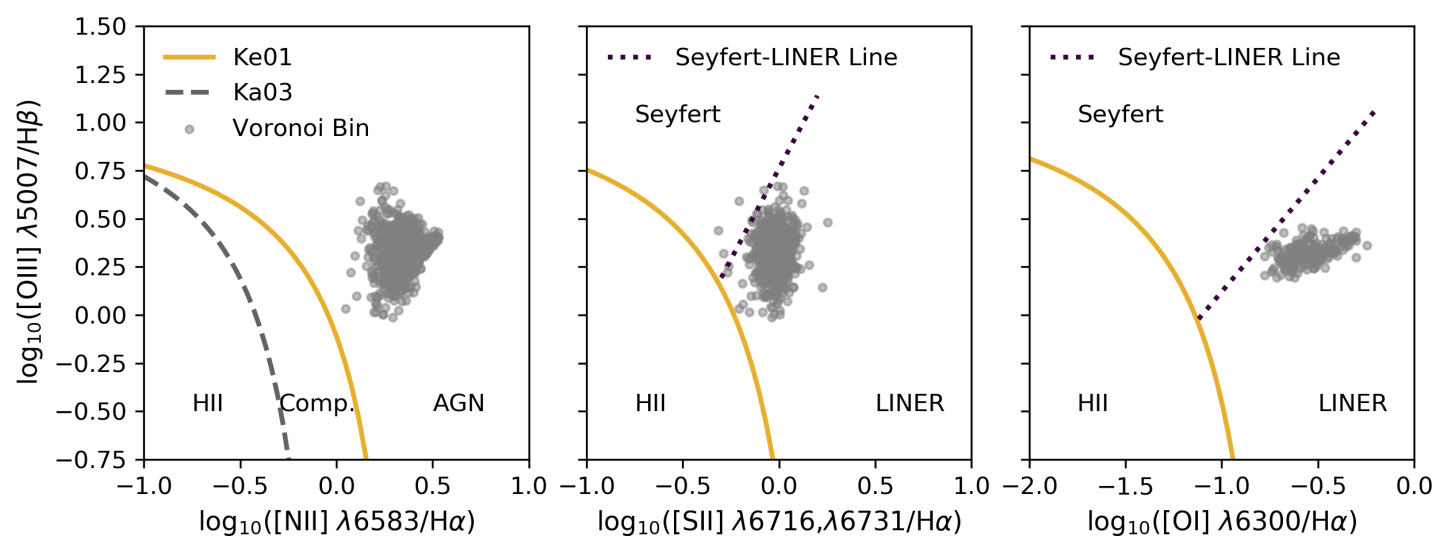

Figure 5. BPT diagrams for IC 1459 . From left to right: $[\mathrm{OIII}] / \mathrm{H} \beta$ vs. $[\mathrm{NII}] / \mathrm{H} \alpha,[\mathrm{OIII}] / \mathrm{H} \beta$ vs. $[\mathrm{SII}] / \mathrm{H} \alpha$, and $[\mathrm{OIII}] / \mathrm{H} \beta$ vs. $[\mathrm{OI}] / \mathrm{H} \alpha$. Each point on the diagnostic diagrams illustrates a Voronoi bin whose emission lines had an $\mathrm{A} / \mathrm{rN}>3$. The Kewley et al. 2001 (Ke01) maximum starburst line appears on each diagnostic diagram (solid, orange line) and separates composite (below) and AGN excitation (above). The Kauffmann et al. 2003 (Ka03) pure SF line (grey dashed line), which appears on the $[\mathrm{OIII}] / \mathrm{H} \beta$ vs. [NII]/H $\alpha$, separates SF excitation (HII; below) and composite excitation (above). The Seyfert-LINER line from Kewley et al. 2006 (dotted line) is in the $[\mathrm{OIII}] / \mathrm{H} \beta$ vs. [SII]/H $\alpha$, and $[\mathrm{OIII}] / \mathrm{H} \beta$ vs. [OI]/ $\mathrm{H} \alpha$ diagnostic diagrams. The Seyfert-LINER line separates spaxels with Seyfert and LINER-like emission. See Section 4.

gas has a filamentary structure, high velocity dispersion $\left(\sigma_{[N I I]}=\right.$ $92 \mathrm{~km} \mathrm{~s}^{-1}$ ) and gas clouds follow non-circular motions. The ionized gas in IC 1459 rotates in the opposite direction than the central stellar component (right column in Figure 3) and is discussed further in Section 6.

The weakest and least abundant emission lines are $\mathrm{H} \beta$ and $[\mathrm{OI}]$ and they appear to exist mostly in the very centre of the galaxy $\left(2^{\prime \prime}\right)$. The detection of these weaker lines is relatively limited by the short exposure of the MUSE cube, poor seeing conditions and high illumination from the Moon during the observations. The [OIII] line is thought to arise from narrow-line regions around the AGN (see Osterbrock 1989). In IC 1459, the [OIII] feature is strongest around the centre, as are most lines, which is an indication of AGN excitation in the central $2^{\prime \prime}$. The strongest and most abundant emission line is [NII] and it exhibits a spiral structure or tidal feature, seen most prominently in the flux map (left column of Figure 3), extending from the galaxy centre to the south, then east, then north region of the MUSE coverage of IC 1459.

In Figure 4, we show images of IC 1459 at progressively smaller radii. We show the gas-rich group environment HI map from Saponara et al. (2018), the optical image from Malin (1985) and the $\mathrm{H} \alpha+[\mathrm{NII}]$ image of IC $1459\left(\sim 1^{\prime} .34\right.$ across) from Goudfrooij et al. (1990). All these have the same orientation as the MUSE maps in Figure 3, and the flux map for [NII] is shown in the plot to the right for comparison. In Goudfrooij et al. (1990) (Figure 4, third panel from left), a clear spiral structure extends from the galaxy centre to the north east (top left) then west (right). This spiral feature sits outside of our MUSE data cube. Our results, however, focus on the central $\sim 1 / 3$ of the image where structure was previously unresolved and the spiral-like structure continues through to the galaxy centre in our MUSE data. The flux in the central region of the map that we present is dominated by [NII]. The image from Goudfrooij et al. (1990) is a composite of $\mathrm{H} \alpha+[\mathrm{NII}]$ which means that regions towards the outskirts could be dominated by $\mathrm{H} \alpha$ as seen in other IFU surveys (e.g., Belfiore et al. 2015). 


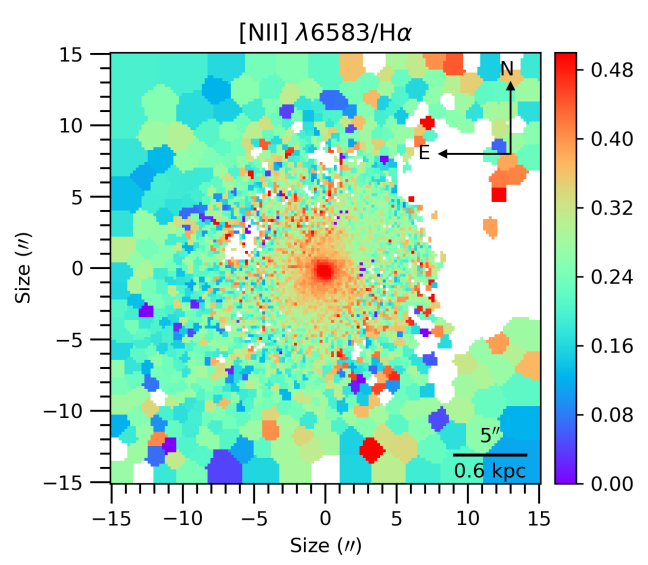

Figure 6. Masked [NII] $\lambda 6583 / \mathrm{H} \alpha$ ratio map of IC 1459 . The central $5^{\prime \prime}$ of the galaxy show higher ratios, indicative of shock excitation. See Section 4.2.

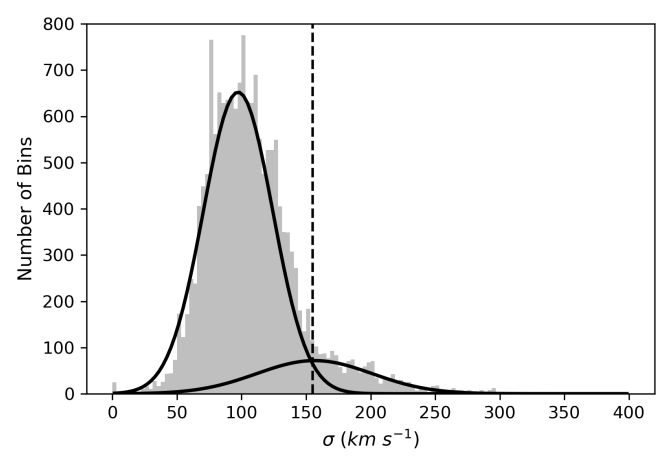

Figure 7. Histogram showing the velocity dispersion of the emission lines with an $\mathrm{A} / \mathrm{rN}>3$. We overlay best-fitting Gaussian curves (solid black lines) and show their intersection at $155 \mathrm{~km} \mathrm{~s}^{-1}$ (dashed black line) that is used to distinguish between the broad and narrow-line components. See Section 4.2

\subsection{Powering Mechanisms of Ionized Gas}

To determine the dominant power source of the emission-line gasses in IC 1459, we created the BPT diagrams (shown in Figure 5; Baldwin et al. 1981; Veilleux \& Osterbrock 1987). Several lines of demarcation appear on these diagrams. Kewley et al. (2001) (Ke01) calculated a theoretical maximum star formation line, which is determined by pure stellar photoionization models' extrenum. In Figure 5, the $\mathrm{Ke} 01$ line appears as the solid, orange line; all data points above $\mathrm{Ke} 01$ are expected to be dominated by AGN activity. Kauffmann et al. (2003) (Ka03) adapted the Ke01 line to include a composite region where both AGN activity and stellar photoionization are power sources for the emission-line profiles. In Figure 5, the $\mathrm{Ka} 03$ line is represented by the dashed, grey line. Kewley et al. (2006) created a new classification, which separates AGN activity from LINER and Seyfert galaxies on the [SII] $\lambda 6716$, $6731 / \mathrm{H} \alpha$ and [OI] $\lambda 6300 / \mathrm{H} \alpha$ BPT diagrams. The Kewley et al. (2006) classification scheme is displayed as the dotted line on Figure 5 .

The BPT diagrams in Figure 5 clearly indicate that the gas is not ionized by young stars. Figure 5 reveals that the gas in IC 1459 has LINER-like emission that could either be attributed to the central nuclear region or to extended low ionization emission regions (LIER) related to IC 1459's evolved stellar population, which is observed frequently in similar galaxies (e.g., Sarzi et al. 2006, 2010; Singh et al. 2013; Belfiore et al. 2016). As the region where this ionization is present is quite large and distant from the nucleus, before making any conclusions we investigate other possible ionization processes. Because the detection of the $\mathrm{H} \beta$ is limited, we spatially resolve the $[\mathrm{NII}] / \mathrm{H} \alpha$ emission-line ratio to identify potential regions of shocked gas (Figure 6). In the $[\mathrm{NII}] / \mathrm{H} \alpha$ map, the ratios are enhanced in the central $2-5^{\prime \prime}$ of IC 1459 , which suggests that shock excitation may be responsible for LINER emission in IC 1459. To look into this further, we utilized the velocity dispersion of the emission-line gas to distinguish shock excitation from AGN activity. We present the histogram of velocity dispersion values in Figure 7. From Figure 7, we can infer that there are at least two physical mechanisms responsible for the ionizing the gas in IC 1459. We characterise these regions by narrow- $\left(\sigma \leq 155 \mathrm{~km} \mathrm{~s}^{-1}\right)$ and broad-linewidths $\left(\sigma>155 \mathrm{~km} \mathrm{~s}^{-1}\right)$ and determined these regions by calculating the point of intersection of the two best-fitting Gaussian curves.

To further understand the ionizing mechanisms in IC 1459, we use the equivalent width of $\mathrm{H} \alpha(\mathrm{W}(\mathrm{H} \alpha)$ ) versus [NII]/H $\alpha$ (WHAN diagram) developed by Cid Fernandes et al. (2011). In Figure 8, $2.5 \%$ of the Voronoi bins have spectra with LINER-like emission. The spaxels with $\mathrm{W}(\mathrm{H} \alpha)<3 \AA$ have emission related neither to AGN nor to starburst activity. Rather, the ionizing mechanism for the gas in these spaxels is most likely from post-asymptotic giant branch (AGB) stars, and can be characterised as LIER-like. From Figure 8, LIER excitation is the dominant powering mechanism in IC 1459 and LINER excitation is mostly limited to the central $2^{\prime \prime}$. This centrally concentrated LINER excitation shows hints of filamentary or jet-like structure. Three bins exhibit Seyfert-like emission, but from their non-centralized and random locations it is evident that these are likely spurious detections and should be ignored.

We speculate that the ionized gas of the narrow component can be explained by the accretion of gas from the intra-group medium, and is therefore the result of tidally disrupted gas from group interactions (Serra et al. 2015; Saponara et al. 2018). This is supported by the extended spiral-like feature we find within the centre of the galaxy and those tidal features found at significantly larger radii in other studies out to gas reservoirs in the larger group environment (Figure 4; Malin 1985; Goudfrooij et al. 1990; Iodice et al. 2020). The ionized gas of the broad line-width component $(\sigma>155 \mathrm{~km}$ $\mathrm{s}^{-1}$ ), however, has two coexisting heating mechanisms according to Figure 8: LINER emission from accretion onto the AGN and LIER emission from post-AGB stars.

One remarkable feature of the accreted ionized gas is that it is counter-rotating with respect to the KDC, which implies that ionized gas is not related to the KDC. A timing argument can be used to support this conclusion. If we assume that the $\mathrm{HI}$ gas in the IC 1459 group is tidally stripped from one of the gas-rich galaxies, then this is likely a relatively recent event. Oosterloo et al. (2018) proposes $2 \mathrm{Gyr}$ as a lower limit. This can be compared with the estimated survival time of $\approx 4$ Gyr of the tidal features around IC 1459 (Mancillas et al. 2019; Iodice et al. 2020), but is significantly younger than the age of the stellar populations found in the KDC, and IC 1459 as a whole (7.6-9.6 Gyr; Prichard et al. 2019). Further evidence that the gas is not related to the formation of the KDC can be established by looking at the stellar orbital distribution of IC 1459 . 

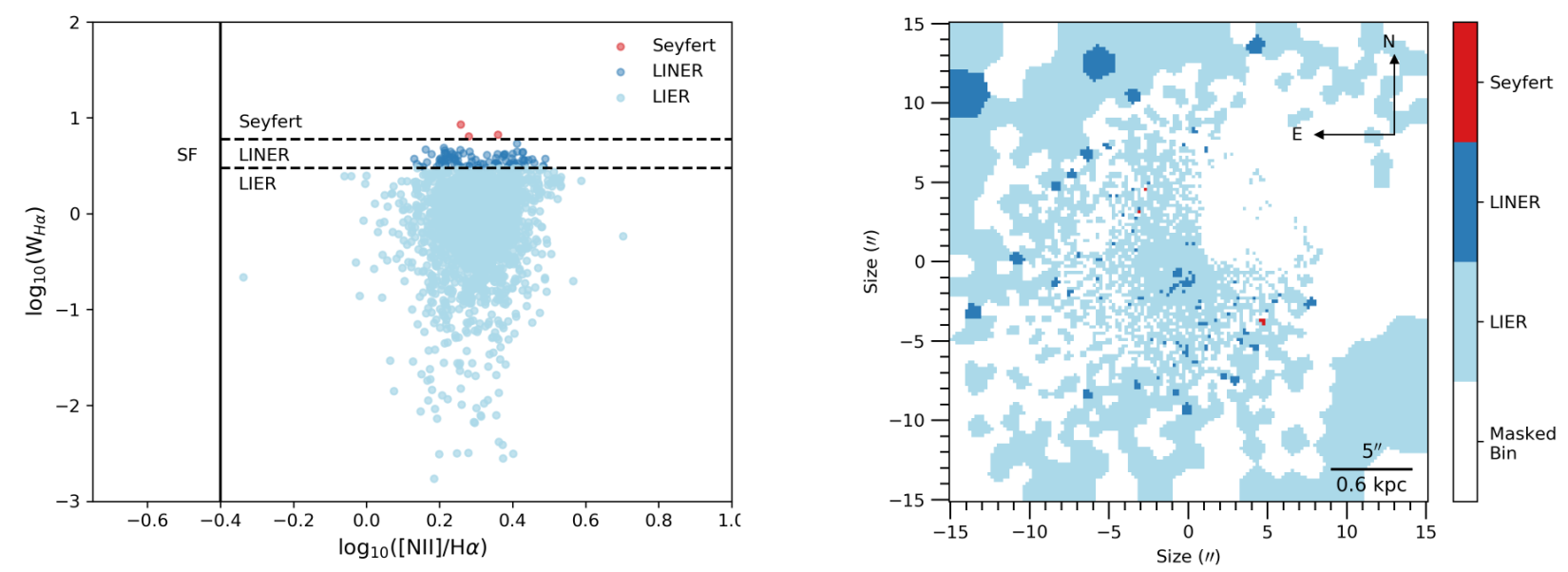

Figure 8. Left: The WHAN diagram of IC 1459. Each point on the diagnostic diagram represents a Voronoi bin.The top most dashed line at W(H $\alpha)=6$ $\AA$ separates Seyfert (above) and LINER (below) excitation and the bottom dashed line at W(H $\alpha)=3 \AA$ separates the LINER-type emission (above) and the LIER-type emission (below). The vertical line at [NII]/H $\alpha=-0.4$ separates starburst (left) and AGN (right) activity. Right: Map showing the spatial location of the ionizing mechanisms determined from the WHAN diagram (left). See Section 4.2.

\section{STELLAR ORBITS WITHIN IC 1459}

In addition to the prominent KDC in the stellar velocity map, MUSE observations of IC 1459 revealed a somewhat unusual feature in the velocity dispersion map (Figure 3, Prichard et al. 2019). Next to the central peak, there is an elongated plateau of high velocity dispersion values, which stretches along the major axis and beyond the visible part of the KDC to about $10^{\prime \prime}$ from the centre. The velocity dispersion is relatively smaller (still above $200 \mathrm{~km} / \mathrm{s}$ ) along the minor axis of the galaxy, while high $(\sim 300 \mathrm{~km} / \mathrm{s})$ along the major axis and subsequently "flares" away from the major axis at radii larger than $10^{\prime \prime}$ (at the edge of the MUSE field). The high velocity dispersion along the major axis is also noticeable on Franx \& Illingworth (1988) long-slit data, but the MUSE maps of Prichard et al. (2019) reveal for the first time the true morphology of the feature (see Figure 1 of Cappellari et al. (2002) for an interpolated velocity dispersion map based on long-slits observations).

Such a velocity feature is reminiscent of the $2 \sigma$ features visible in the velocity dispersion maps of some disk galaxies (Krajnović et al. 2011). They are characterised with two symmetric peaks along the major axis, which arise as these galaxies are made of two counter-rotating disks (e.g. NGC4550, as a prototypical example of the class; Rix et al. 1992; Rubin et al. 1992; Emsellem et al. 2004). This suggests that IC 1459 could also have strong counter-rotating components, related to the KDC. IC 1459 is not a disk galaxy, nor it is likely made of two counter-rotating disks. Nevertheless, it is analogous to another giant elliptical, NGC 5813, which has a similar high velocity dispersion plateau (Krajnović et al. 2015). Dynamical models showed that stellar kinematics of NGC 5813 can be reproduced by two counter-rotating orbital families, one of which is dominant in the centre and is responsible for the KDC, while at larger radii the two families contribute with similar masses, cancelling out the net rotation, but increasing the line width (dispersion) along the major axis (Krajnović et al. 2015). The similarities between the kinematics of IC 1459 and NGC 5813 are striking, and we set up dynamical models to investigate the internal structure of IC 1459.

We constructed axisymmetric Schwarzschild models based on the orbit superposition approach (Schwarzschild 1979) using the Leiden code (Cappellari et al. 2006). Based on an assumption on the gravitational potential, the code builds a library of orbits, which are then combined to reproduce the observed stellar distribution and kinematics. Axisymmetric gravitational potentials support only one family of stable orbits, the short axis tubes (de Zeeuw 1985), which are defined by three integrals of motion: energy $(E)$, the short axis projection of the angular momentum $\left(L_{z}\right)$, and the third (non-analytical) integral $\left(I_{3}\right)$. This galaxy was modelled already with an earlier version of the code (Cappellari et al. 2002), but there are some important differences: here we use an evolved version of the code adapted for integral-field data (in terms of the orbital set up) and a much higher number of orbits, as specified above. The biggest difference, however, is in the MUSE stellar kinematics used to constrain the models. The details of Schwarzschild modelling and our set up are given in Appendix A.

In Figure 9 we show the main result of the modelling: meridional planes of the galaxy, represented by polar grids of orbital starting position (white dots), each panel is for a given energy (represented with the radius of the circular orbit at that energy shown in green). Each panel is divided into right and left sides, corresponding to prograde and retrograde orbits, respectively. The definition of what is prograde and retrograde is arbitrary, and we assign the component responsible for the KDC as the prograde component ("+" sign). High angular momentum orbits are found in the lower right and left corners of the panels. Orbits with increasing $I_{3}$ values are found higher along the $y$-axis. The colour contours show the weights assigned by the model to the orbits. Not all orbits are selected (black regions), and there are regions in the meridional plane which are given more weight.

The conclusion is that the model requires both prograde and retrograde orbits throughout the galaxy body, each component having essentially the same fraction of the total mass (51:49 per cent split, within the extent of the MUSE data). Within the KDC (for $r<5^{\prime \prime}$ ) the ratio of the prograde to retrograde orbits is approximately 58:42 per cent, this is followed by a transition region between $6^{\prime \prime}$ and $12^{\prime \prime}$ with the orbital split of 50:50, while at the large radii $\left(r>12^{\prime \prime}\right)$ this ratio reverses to 43:57. Therefore, the KDC in IC 




Figure 9. Orbital space of the axisymmetric Schwarzschild model of IC 1459 represented with weights (in colour) assigned to orbits that bulid the model. Each orbit is defined by three integrals of motion: energy $(E)$, projections of the angular momentum along the short axis $\left(L_{z}\right)$ and the non-analytic third integral. Each hemisphere plots the meridional plane $(R, z)$ for a given energy, where the white dots indicate the initial positions of orbits. Green numbers in the upper-left corner show the radius (in arcsec) of the circular orbit of a given energy. Within each hemisphere, the angular momentum changes along the $x$-axis while the value of the third integral varies along the $y$-axis. Prograde orbits are shown on the right side of each panel (indicated with a " + " sign), while the retrograde orbits are shown on the left side ("-" sign). The ratios of the total mass assigned to prograde and retrograde orbits are printed above the corresponding side of the panel. We show only a subset of radii, representative of the observed stellar kinematics. The relative colour coding of the orbital mass weights is shown with the colourbar, where 1 represents the largest mass weight assigned to an orbit at the given energy.

1459 is not a separate entity residing in the centre, kinematically decoupled from the rest of the galaxy. On the contrary, the KDC is an integral part of the galaxy made up of orbits belonging to a single family of short axis tubes that rotate in the prograde sense, and extend from the central parts to large scales. There is another family of short axis orbits which also extend throughout the system, but rotate in the retrograde sense. The morphology of the velocity map (the KDC and its counter-rotation with respect to the large scales) can be explained through a superposition of these two orbital families, where the prograde dominates in the central parts $\left(<5-10^{\prime \prime}\right)$, while the retrograde in the outer parts $\left(>5-10^{\prime \prime}\right)$.

Cappellari et al. (2002) also presented the orbital structure of their best fit model, which bears many similarities to our model. They do not provide details on the mass fractions of the prograde and retrograde orbital families, but the model implies a more localised presence of the prograde orbits, which are mostly restricted to the KDC region. The difference in the results between their and our work originates essentially from the improved quality of our data.

The continuous distribution of both prograde and retrograde orbits within the area covered by the MUSE data also supports conclusions of stellar population analysis from Prichard et al. (2019): IC 1459 has relatively constant initial mass function (IMF) and metallicity, the galaxy comprises a fairly homogeneous stellar population, and the apparent counter-rotating core is due to a slight mass imbalance of stars on orbits.

From a dynamical point of view, the two orbital families have to come from different progenitors of similar masses. This major merger formed the central regions (observed by MUSE) of the galaxy and is responsible for the observed stellar kinematics. The emission-line gas might have a similar angular momentum vector as the retrograde component that is dominating the outer parts of the galaxy, but this is not sufficient to associate it to the formation of this or any other stellar component. Based on the timing argument for the accretion of gas, the old stellar populations, as well as taking into account the equal masses of the prograde and retrograde components, the emission-line gas is not related to the formation of stars making up the current stellar distribution. Therefore, the ionized gas in IC 1459 must come from a later-stage accretion.

\section{DISCUSSION AND CONCLUSION}

In this work we have investigated the origin of ionized gas in IC 1459, a massive, slow-rotator ETG with a rapidly counter-rotating stellar core. We used IFU data from MUSE to study spatially resolved structure, flux, and kinematics of the gas emission lines. We also investigated the orbital space of the stellar populations through Schwarzschild modelling. This combined study of both the ionized gas and stars in IC 1459 builds on previous work to construct a more complete evolutionary history of the galaxy.

We used the Voronoi binning method of Cappellari \& Copin (2003) to adaptively bin each spaxel over the MUSE cube to a roughly constant signal to noise ( $\mathrm{S} / \mathrm{N}>20)$. Using pPXF, we extracted the stellar and gas information from the MUSE data cube and assessed their spatially resolved structure. We determined the dominant power source of emission-line gasses in IC 1459 by evaluating emission-line gas maps, diagnostics, and velocity dispersions. We also inspected the stellar orbital distribution through Schwarzchild modelling. Our key findings are outlined below.

(i) We present the first detailed flux and kinematic maps of the ionized gas in the central $30^{\prime \prime} \times 30^{\prime \prime}$ of IC 1459 . The gas is blue shifted to the south east with a maximum relative velocity of -344 $\mathrm{km} \mathrm{s}^{-1} \pm 9$ and red shifted to the northwest with a maximum relative velocity of $389 \mathrm{~km} \mathrm{~s}^{-1} \pm 7$. The gas in the central $2^{\prime \prime}$ of the galaxy has disordered, dispersion-dominated kinematics.

(ii) The least abundant emission lines are $\mathrm{H} \beta$ and $[\mathrm{OI}]$ and we find these exist mostly in the central $2^{\prime \prime}$ of the galaxy. The strongest, most abundant emission line is [NII], which exhibits an extended disk-like structure or tidal feature within the MUSE coverage of IC 1459. The structure of the [OIII], $\mathrm{H} \alpha$ and [SII] emission-line gasses appears to be in a spiral-like structure along the galaxy's major axis.

(iii) We find that LINER and LIER excitation are the two domi- 
nant ionizing mechanisms in IC 1459. By investigating the velocity dispersion, emission-line ratios and the WHAN diagram of the emission-line gas, we determined that the ionization mechanisms could have contributions from shock heating and AGN excitation. However, given that LIER-like emission in IC 1459 is extended (kpc scale), the gas is most likely heated by evolved AGB stars within the old stellar core rather than a central nuclear source. This result adds to a growing body of literature that extended LIER emission is from pAGB stars rather than a central, low luminosity AGN (e.g.; Sarzi et al. 2006; Singh et al. 2013; Belfiore et al. 2016).

(iv) The central structure of ionized gas is rotating in the opposite direction to the central stellar component, suggesting that the origin of the ionized gas is different than that of the central stellar component. Although it is likely that IC 1459 has undergone at least one gas-rich merger in its past, we do not believe the ionized gas currently present in IC 1459 is from a major merger event with a spiral galaxy from the group of galaxies surrounding IC1459. Within the IC 1459 galaxy group, there is evidence for a large (500 kpc-long; Oosterloo et al. 2018), massive $\left(10^{10} \mathrm{M}_{\odot}\right.$; Iodice et al. 2020) HI debris complex near IC 1459 (Saponara et al. 2018). We propose that the ionized gas that we detect in the central $30^{\prime \prime} \times$ $30^{\prime \prime}$ of IC 1459 is from the ongoing, late-stage accretion from the group environment. Given the extent of the HI debris complex, the velocity dispersion of the IC 1459 group $\left(223 \pm 62 \mathrm{~km} \mathrm{~s}^{-1}\right.$; Brough et al. 2006), along with simulations from Mancillas et al. (2019), the accretion onto IC 1459 has been occurring for the last $\approx 2-4$ Gyrs (Figure 4; Oosterloo et al. 2018; Iodice et al. 2020).

(v) The stellar content of IC 1459 is evenly split into a prograde and a retrograde component. The prograde component is somewhat more centrally concentrated, while the retrograde component dominates at larger scales. Their (luminosity weighted) superposition is responsible for the complex appearance of the velocity and velocity dispersion maps. This also means that the origin of the KDC in IC 1459 is linked to an early major merger, and is not related to more recent interaction events, evident in the tidal features and the abundance of the inter-group gas.

\section{ACKNOWLEDGEMENTS}

We thank the anonymous referee for valuable comments on the paper. CRM and RAJ gratefully acknowledge support by NSF REU grant AST-1757321, the Massachusetts Space Grant, and by the Nantucket Maria Mitchell Association.

\section{DATA AVAILABILITY}

The reduced MUSE data that support the findings of this study are available in the ESO Science Archive at http://archive. eso.org/cms.html and can be accessed with program ID 094.B0298(A).

\section{REFERENCES}

Alatalo K., et al., 2011, ApJ, 735, 88

Annibali F., Bressan A., Rampazzo R., Zeilinger W. W., Vega O., Panuzzo P., 2010, A\&A, 519, A40

Bacon R., et al., 2001, MNRAS, 326, 23

Bacon R., et al., 2010, in Ground-based and Airborne Instrumentation for Astronomy III. p. 773508, doi:10.1117/12.856027

Baldwin J. A., Phillips M. M., Terlevich R., 1981, PASP, 93, 5
Barrera-Ballesteros J. K., et al., 2015, A\&A, 582, A21

Belfiore F., et al., 2015, MNRAS, 449, 867

Belfiore F., et al., 2016, MNRAS, 461, 3111

Bender R., 1988, A\&A, 202, L5

Brough S., Forbes D. A., Kilborn V. A., Couch W., 2006, MNRAS, 370, 1223

Buson L. M., et al., 1993, A\&A, 280, 409

Caldwell N., 1984, Publications of the Astronomical Society of the Pacific, 96, 287

Cappellari M., 2016, ARA\$\&\$A, 54, 597

Cappellari M., 2017, MNRAS, 466, 798

Cappellari M., Copin Y., 2003, MNRAS, 342, 345

Cappellari M., Emsellem E., 2004, PASP, 116, 138

Cappellari M., Verolme E. K., van der Marel R. P., Verdoes Kleijn G. A., Illingworth G. D., Franx M., Carollo C. M., de Zeeuw P. T., 2002, ApJ, 578,787

Cappellari M., et al., 2006, MNRAS, 366, 1126

Cappellari M., et al., 2011, MNRAS, 413, 813

Cappellari M., et al., 2013, MNRAS, 432, 1862

Carollo C. M., Danziger I. J., 1994, MNRAS, 270, 523

Cenarro A. J., Cardiel N., Gorgas J., Peletier R. F., Vazdekis A., Prada F., 2001, MNRAS, 326, 959

Cheung E., et al., 2016, Nature, 533, 504

Cid Fernandes R., Stasińska G., Mateus A., Vale Asari N., 2011, MNRAS, 413,1687

Davis T. A., et al., 2011, MNRAS, 417, 882

Efstathiou G., Ellis R. S., Carter D., 1982, MNRAS, 201, 975

Emsellem E., et al., 2004, MNRAS, 352, 721

Emsellem E., et al., 2007, MNRAS, 379, 401

Emsellem E., et al., 2011, MNRAS, 414, 888

Farage C. L., McGregor P. J., Dopita M. A., Bicknell G. V., 2010, ApJ, 724, 267

Ferrarese L., Merritt D., 2000, ApJL, 539, L9

Forbes D. A., Reitzel D. B., Williger G. M., 1995, AJ, 109, 1576

Franx M., Illingworth G. D., 1988, ApJL, 327, L55

Goudfrooij P., Norgaard-Nielsen H. U., Hansen L., Jorgensen H. E., de Jong T., 1990, A\&A, 228, L9

Goudfrooij P., Hansen L., Jorgensen H. E., Norgaard-Nielsen H. U., 1994, A\&AS, 105, 341

Ho I. T., et al., 2016, MNRAS, 457, 1257

Huchra J. P., Geller M. J., 1982, ApJ, 257, 423

Iodice E., et al., 2020, A\&A, 635, A3

Jedrzejewski R., Schechter P. L., 1988, ApJ, 330, L87

Kauffmann G., et al., 2003, MNRAS, 346, 1055

Kewley L. J., Dopita M. A., Sutherland R. S., Heisler C. A., Trevena J., 2001, ApJ, 556, 121

Kewley L. J., Groves B., Kauffmann G., Heckman T., 2006, MNRAS, 372, 961

Kim D.-W., 1989, ApJ, 346, 653

Krajnović D., et al., 2008, MNRAS, 390, 93

Krajnović D., et al., 2011, MNRAS, 414, 2923

Krajnović D., et al., 2015, MNRAS, 452, 2

Macchetto F., Pastoriza M., Caon N., Sparks W. B., Giavalisco M., Bender R., Capaccioli M., 1996, A\&AS, 120, 463

Malin D. F., 1985, in Nieto J.-L., ed., Lecture Notes in Physics, Berlin Springer Verlag Vol. 232, New Aspects of Galaxy Photometry. pp 2732, doi:10.1007/BFb0030917

Mancillas B., Duc P.-A., Combes F., Bournaud F., Emsellem E., Martig M., Michel-Dansac L., 2019, A\&A, 632, A122

Monreal-Ibero A., Arribas S., Colina L., 2006, ApJ, 637, 138

Monreal-Ibero A., Arribas S., Colina L., Rodríguez-Zaurín J., AlonsoHerrero A., García-Marín M., 2010, A\&A, 517, A28

Naab T., et al., 2014, MNRAS, 444, 3357

Oosterloo T. A., Zhang M. L., Lucero D. M., Carignan C., 2018, arXiv e-prints, p. arXiv:1803.08263

Osterbrock D. E., 1989, Astrophysics of gaseous nebulae and active galactic nuclei

Paturel G., et al., 1997, A\$\&\$AS, 124, 109 
Phillips M. M., Jenkins C. R., Dopita M. A., Sadler E. M., Binette L., 1986, AJ, 91, 1062

Prandoni I., Laing R. A., de Ruiter H. R., Parma P., 2012, in Journal of Physics Conference Series. p. 012067, doi:10.1088/17426596/372/1/012067

Prichard L. J., Vaughan S. P., Davies R. L., 2019, MNRAS, 488, 1679

Rich J. A., Dopita M. A., Kewley L. J., Rupke D. S. N., 2010, ApJ, 721, 505

Rich J. A., Kewley L. J., Dopita M. A., 2011, ApJ, 734, 87

Rix H.-W., Franx M., Fisher D., Illingworth G., 1992, ApJ, 400, L5

Rubin V. C., Graham J. A., Kenney J. D. P., 1992, ApJ, 394, L9

Samurović S., Danziger I. J., 2005, MNRAS, 363, 769

Sánchez-Blázquez P., et al., 2006, MNRAS, 371, 703

Saponara J., Koribalski B. S., Benaglia P., Fernández López M., 2018, MNRAS, 473, 3358

Sarzi M., et al., 2006, MNRAS, 366, 1151

Sarzi M., et al., 2010, MNRAS, 402, 2187

Schwarzschild M., 1979, ApJ, 232, 236

Serra P., et al., 2015, MNRAS, 452, 2680

Sharples R., et al., 2013, The Messenger, 151, 21

Singh R., et al., 2013, A\&A, 558, A43

Tingay S. J., Edwards P. G., 2015, MNRAS, 448, 252

Vazdekis A., Sánchez-Blázquez P., Falcón-Barroso J., Cenarro A. J., Beasley M. A., Cardiel N., Gorgas J., Peletier R. F., 2010, MNRAS, 404, 1639

Vazdekis A., Koleva M., Ricciardelli E., Röck B., Falcón-Barroso J., 2016, MNRAS, 463, 3409

Veilleux S., Osterbrock D. E., 1987, ApJS, 63, 295

Verdoes Kleijn G. A., van der Marel R. P., Carollo C. M., de Zeeuw P. T., 2000, AJ, 120, 1221

Weijmans A.-M., et al., 2014, MNRAS, 444, 3340

de Zeeuw P. T., 1985, MNRAS, 216, 273

de Zeeuw P. T., et al., 2002, MNRAS, 329, 513

van der Marel R. P., Cretton N., de Zeeuw P. T., Rix H., 1998, ApJ, 493, 613

\section{APPENDIX A: A SCHWARZSCHILD MODEL OF IC1459}

We use the Leiden version of the Schwarzschild orbital superposition code as presented by Cappellari et al. (2006). The Schwarzschild codes are typically used to determine the masses of supermassive black holes in the centres of galaxies, but in this case we are only interested in the global distribution of orbits in IC1459. As mentioned in the main text, there is only one orbital family, short axis tubes, which can rotate in the prograde and retrograde sense within an axisymmetric potential well. Each orbit is defined by three integrals of motion: energy $(E)$, the short axis projection on the angular momentum $\left(L_{z}\right)$, and the third integral $\left(I_{3}\right)$. The code samples the orbits on 41 energy points, which are radially spaced throughout the galaxy with a logarithmic step. In addition, for each radius defined by the energy step, there are a further 11 angular and 11 radial points, arranged in a polar grid, which sample the combination of $L_{z}$ and $I_{3}$. The orbits are also dithered (6 dithers in each dimension), so the final numbers of orbits superseeds 2.1 million (for details see Cappellari et al. 2006). In order to ensure a smooth distribution of orbital weights we apply a moderate regularisation $(\Delta=4$, see van der Marel et al. 1998), but we verified that the results did not differ significantly when the regularisation was not used.

As we are interested only in the internal orbital structure we use some of the input parameters from Cappellari et al. (2002), which modeled IC1459 to estimate the mass of the supermassive black hole. We use their parameterisation of stellar light (their Table 4), inclination $(90 \mathrm{deg})$, mass of the central black hole $\left(\mathrm{M}_{B H}=\right.$ $\left.2.6 \times 10^{9} \mathrm{M}_{\odot}\right)$, and the stellar mass to light ratio $(\mathrm{M} / \mathrm{L}=3)$. In this way, the gravitational potential is fully specified and only the MUSE kinematics are used to constrain which orbits will end up in the final model representing the galaxy.

IC1459 has a small photometric twist and, being a giant elliptical and a massive slow rotator, it is likely that it is at least weakly triaxial (Weijmans et al. 2014). However, the central kinematics covered by MUSE are largely bisymetric with respect to the (projected) minor axis of the galaxy, although a kinematic twist is visible towards the edge of the field. Nevertheless, as we focus on the central regions, we follow Cappellari et al. (2002), and apply the axisymmetric code as an approximate dynamical models (similar to assumptions for modelling NGC 5813 by Krajnović et al. 2015). As the models are by construction axisymmetric, we symmetrise the MUSE kinematics using point-(anti)symmetry around the photometric minor axis $\left(\mathrm{PA}=123^{\circ}\right)$, averaging the values at positions: $[(x, y),(x,-y),(-x, y),(-x,-y)]$, but keeping the original errors for each point.

In Figure A1 we show the quality of our Schwarzschild model in the sense of the kinematic data-model prediction comparison. The bottom row in the figure shows the model-data residuals, divided by the errors. The model is able to reproduce the features on the velocity and the velocity dispersion maps well, as well as features on odd Gauss-Hermite coefficient maps. The biggest and systematic discrepancies are found for $h_{4}$ and $h_{6}$ maps where model overpredicts the data; they are however within $2 \sigma$ uncertainty level except in a few bins towards the edges of the field.

This paper has been typeset from a $\mathrm{T}_{\mathrm{E}} \mathrm{X} / \mathrm{LT}_{\mathrm{E}} \mathrm{X}$ file prepared by the author. 

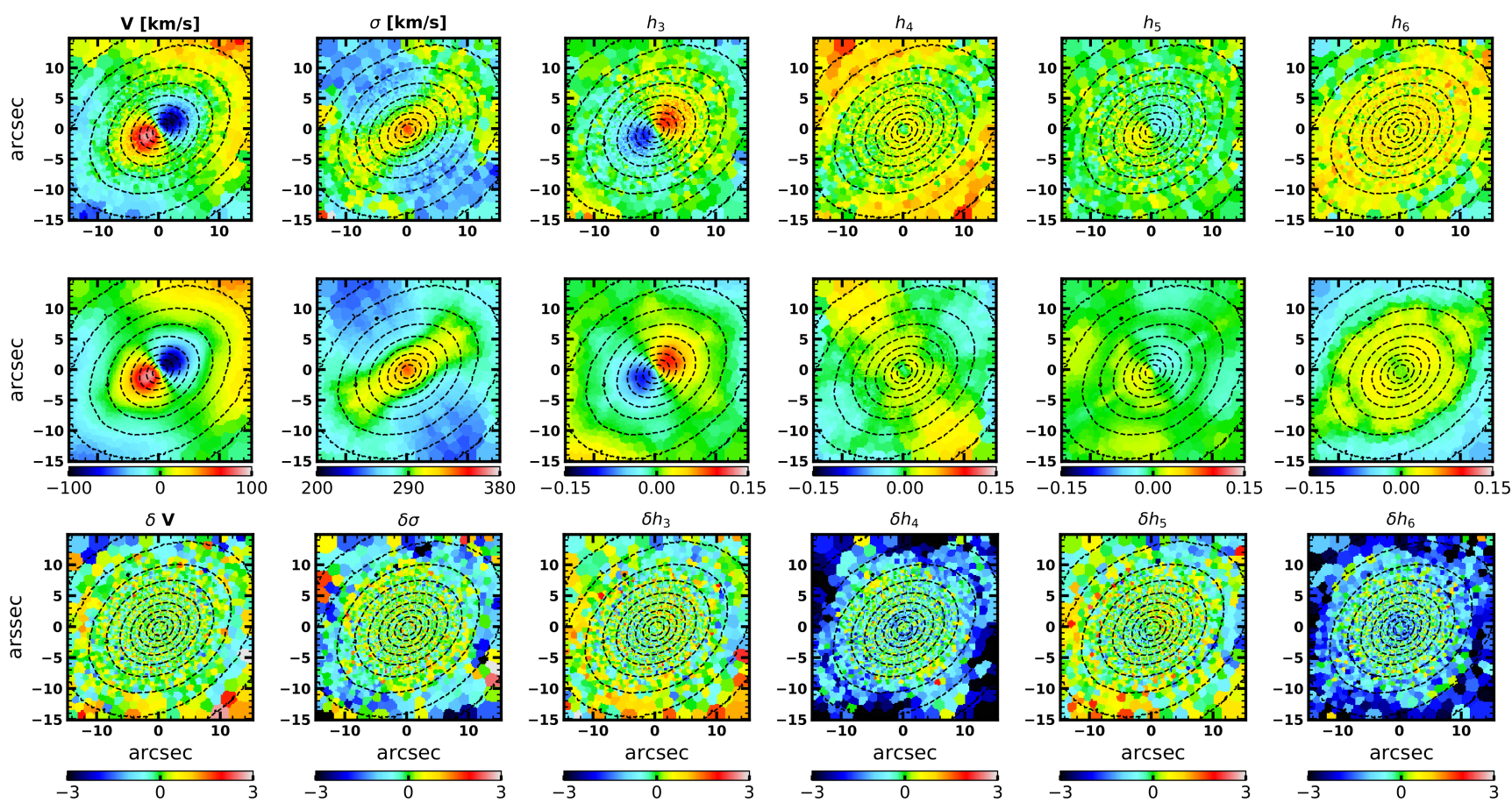

Figure A1. Comparison between the kinematic data and the result of the Schwarzschild modelling. Top row: Symmetrised MUSE stellar kinematics, starting from left to right: the mean velocity, the velocity dispersion, and the Gauss-Hermite coefficients $h_{3}, h_{4}, h_{5}$ and $h_{6}$. Middle row: kinematics of the Schwarzschild dynamical model, showing the same maps as in the top row. Bottom row: Residuals between the Schwarzschild models and the symmetrised MUSE kinematics, divided by the observational error. 